\title{
DESARROLLO DE UN NANOSENSOR APTAMERICO BASADO EN NANOTRIANGULOS DE ORO PARA LA DETECCIÓN DE AFLATOXINA B1 MEDIANTE LSPR
}

\author{
NANOAPTASENSOR DEVELOPMENT BASED ON GOLD \\ NANOTRIANGLES FOR THE DETECTION OF AFLATOXIN B1 BY LSPR
}

\author{
Lorena Veliz-Portal ${ }^{1}$, Mary Licuona-Puma' ${ }^{1}$, Sara Córdova-Tuppia' ${ }^{1}$ \\ Yulán Hernández-García ${ }^{1}$, Betty Galarreta-Asian ${ }^{1 *}$
}

${ }^{1}$ Sección Química, Pontificia Universidad Católica del Perú, Departamento de Ciencias. Lima, Perú.

Recibido (Recieved): 29/11/2019 Aceptado (Accepted): 22/01/2020

\begin{abstract}
RESUMEN
La aflatoxina B1 (AFB1) es una micotoxina producida por la especie de hongos Aspergillus que afecta a una gran variedad de productos agrícolas como la kiwicha. La AFB1 es conocida por ser nefrotóxica, hepatotóxica y carcinogénica, lo que ha llevado a una estricta regulación en todo el mundo y a la necesidad de tener nuevos métodos que permitan su detección rápida, sencilla y selectiva. Los nanosensores aptaméricos basados en nanotriángulos de oro (AuNTs) surgen como una alternativa simple y eficaz a los sistemas tradicionales de detección. Por una parte, los aptámeros hacen que el sistema sea estable y muy selectivo debido a su gran afinidad, pero a un bajo costo y, por otro, las excepcionales propiedades ópticas de las nanopartículas de oro permiten implementar el sensor en una técnica analítica sencilla como la espectroscopía UV-Vis-NIR. Esto se debe a la resonancia de plasmón superficial localizado (LSPR), que permite monitorear la interacción entre el aptámero y la toxina y relacionar los cambios espectrales con el contenido de micotoxina. Los nanosensores aptaméricos desarrollados en esta investigación fueron evaluados en diferentes solventes y tampones, siendo el mejor de ellos el de PBS pH 7.5 $\left(R^{2}=0.98101\right)$ logrando detectar AFB1 en el rango de 0-780 ppbs. Adicionalmente, el análisis multivariante por regresión de mínimos cuadrados parciales (PLS) de los espectros logró obtener un límite de detección de 22 ppbs. Finalmente, los nanosensores han demostrado ser lo suficientemente robustos para obtener buenos resultados aun en matrices complejas como extractos de kiwicha.
\end{abstract}

Palabras Clave: Aflatoxina, nanosensores, oro, micotoxinas, aptámeros.

\begin{abstract}
Aflatoxin B1 (AFB1) is one of the most frequent mycotoxins produced by certain species of the fungi Aspergillus, which affects a wide variety of agricultural products such as kiwicha. AFB1 is known to be nephrotoxic, hepatotoxic and carcinogenic, and has led to strict regulation throughout the world and the need to have new rapid, simple and selective methods to detect the mycotoxin. The nanoaptasensors based on gold nanotriangles (AuNTs) emerge as a simple and effective alternative to traditional detection systems. On the one hand, aptamers make the system stable and very selective due to its high affinity, but at a low cost. On the other hand, the exceptional optical properties of gold nanoparticles allow the implementation of the sensor in a simple analytical technique such as UV-Vis-NIR spectroscopy. This development is possible due to the localized surface plasmon resonance (LSPR), which allows monitoring the interaction between the aptamer and the toxin and correlating the spectral changes with the mycotoxin content. The sensitivity of the nanosensors was evaluated in different solvents and buffers within the 0-780 ppbs AFB1 range. Best results were obtained using PBS buffer with $\mathrm{MgCl}_{2} \mathrm{pH} 7.5\left(\mathrm{R}^{2}=\right.$ 0.98101). Furthermore, the spectral analysis by the partial least squares regression (PLS) method it was possible to achieve a detection limit of 22 ppbs. Finally, the developed nanoaptasensors have shown good responses even in the presence of a complex matrix such as kiwicha.
\end{abstract}

Keywords: Aflatoxin, nanosensors, gold, micotoxin, aptamers.

\footnotetext{
* Corresponding author.:
}

E-mail: bgalarreta@pucp.pe 


\section{INTRODUCCION}

Las micotoxinas son metabolitos secundarios producidos por una amplia variedad de hongos, que pueden resultar peligrosas para los seres vivos a través de la inhalación, ingesta o contacto con la piel [1], [2]. Dentro del variado grupo de micotoxinas, uno de los más relevantes es el de las aflatoxinas. Estos compuestos son derivados difuranocumarínicos provenientes de la especie Aspergillus y pueden encontrarse en cereales, granos, nueces y otros suplementos alimenticios y su proliferación puede darse en cualquier etapa de la cadena productiva, siendo reconocidas como contaminantes naturales de la comida. Actualmente, la aflatoxina B1 (AFB1) es considerada una de las más tóxicas debido a que puede causar severos daños hepáticos y nefrológicos, además de ser un potente agente carcinogénico [3], [4].

Debido al gran número de enfermedades que puede causar la AFB1, los organismos de salud internacionales han establecido el límite máximo en el rango de los ppbs [5]. Por esta razón, se han desarrollado innumerables métodos, basados en técnicas cromatográficas, capaces de detectar AFB1 en matrices biológicas complejas. Entre los métodos más utilizados se encuentran LC-MS/MS y GC/MS pues ofrecen una gran sensibilidad y selectividad; sin embargo, ambos necesitan de largos tiempos de preparación y complejos procesos de extracción de muestras [6], personal altamente capacitado y equipos sofisticados.

Además de los métodos cromatográficos, uno de los ensayos más utilizados para la detección de AFB1 son las pruebas de ELISA, debido a su amplia disponibilidad, sencillez y al gran número de muestras que pueden ser analizadas. A pesar de ello, este tipo de ensayos se ven limitados pues requieren del uso de anticuerpos, los cuales suelen tener baja estabilidad y se degradan fácilmente ante variaciones de temperatura y $\mathrm{pH}$, además de ser de un único uso [3], [4], [7].

Frente a las desventajas de los métodos tradicionales, una de las nuevas alternativas para la identificación y cuantificación de toxinas es el uso de aptámeros. Estas cadenas cortas de oligonuleótidos sintéticos biomimetizan a los anticuerpos y son capaces de reconocer con una alta especificidad a un analito en particular. Sin embargo, a diferencia de estos, los aptámeros son más estables y altamente reproducibles, por lo que están siendo cada vez más utilizados para la detección de toxinas [2]. Además, el hecho de que los aptámeros se sinteticen artificialmente hace que no sólo su costo sea menor, sino que puedan introducirse las modificaciones deseadas de manera sencilla (grupos tiol, fluoróforos, biotina, etc.) [8], [9].

Por otro lado, las nanopartículas metálicas como los nanotriángulos de oro (AuNTs) poseen propiedades ópticas especiales que permiten el desarrollo de sensores accesibles y sensibles aplicados a la seguridad alimentaria y a la salud pública [10]-[12]. Una de estas propiedades ópticas es la resonancia de plasmón superficial localizado (LSPR), cuya banda característica depende del tamaño, forma, material y del ambiente próximo que rodea a la nanopartícula [13]. En el caso de las nanopartículas de metales nobles, los cambios en la banda LSPR se suelen monitorear en el rango UV-Vis-NIR y se pueden relacionar con el contenido del analito [13], [14].

En esta investigación se presenta el desarrollo de un nanosensor aptamérico basado en AuNTs para la detección y cuantificación de AFB1 utilizando LSPR. La secuencia de aptámero fue seleccionada a partir de estudios previos realizados [15] y donde se añadió un extremo tiolado. Además, los nanosensores aptaméricos fueron evaluados con diferentes solventes y tampones reportados en la literatura [16] para su optimización, y se demostró la robustez del sistema al evaluar la respuesta en extractos de muestras reales de kiwicha contaminadas con AFB1.

\section{MATERIALES Y METODOS}

\subsection{REACTIVOS}

Todas las soluciones fueron preparadas utilizando agua ultrapura. Metanol y acetonitrilo (grado HPLC), $\mathrm{CaCl}_{2}, \mathrm{HAuCl}_{4}, \mathrm{KCl}, \mathrm{KH}_{2} \mathrm{PO}_{4}, \mathrm{~K}_{2} \mathrm{HPO}_{4}, \mathrm{MgCl}_{2}$, ácido mercaptopropiónico (MPA), $\mathrm{NaCl}, \mathrm{Na}_{2} \mathrm{~S}_{2} \mathrm{O}_{3}$, tris(hidroximetil)aminometano (TRIS), dodecilsulfato de sodio (SDS) y AFB1 fueron adquiridos en Sigma Aldrich (USA). El oligonucleótido de secuencia 5'- GTTGGG-CAC-GTG-TTG-TCT-CTC-TGT-GTC-TCG-TGC-CCT-TCGCTA-GGC-CC-(CH2)6-SH 3' fue adquirido de Eurogentec (Bélgica). Las jeringas con filtro de fibra de vidrio fueron adquiridas de Neogen (USA).

\subsection{EQUIPOS}

La caracterización de los nanosensores se realizó mediante el uso de un espectrofotómetro UV-Vis-NIR (Genesys 10S, Thermo Scientific-USA) y un microscopio electrónico de transmisión (TEM) (LVEM5, Delong Instruments - República Checa). La funcionalización del sensor se utilizó una centrífuga (Sigma 1-16, Alemania), mientras que para la preparación de los extractos de kiwicha se empleó una centrifuga refrigerada (5430R, Eppendorf - USA). Finalmente, se utilizaron también, una incubadora (MyTemp mini H2200-HC, Benchmark 
Scientific - USA), agitadores (MVOR-03, SBS-USA) y un baño de ultrasonido (97043-930, VWR-USA)

\subsection{SíNTESIS DE NANOTRIÁNGULOS DE ORO (AUNTS)}

Los nanotriángulos de oro (AuNTs) fueron sintetizados usando el protocolo descrito por Pelaz y col. con algunas modificaciones [17], [18]. En primer lugar, se realizó una primera adición de $\mathrm{Na}_{2} \mathrm{~S}_{2} \mathrm{O}_{3}$ (8.75 mL, $0.5 \mathrm{mM}$ ) con una bomba de jeringa (flujo 9 $\mathrm{mL} / \mathrm{min})$ a una solución acuosa de $\mathrm{HAuCl}_{4}(5 \mathrm{~mL}, 2$ $\mathrm{mM}$ ) bajo agitación mecánica constante. Luego de 9 minutos, se hizo una segunda adición de $\mathrm{Na}_{2} \mathrm{~S}_{2} \mathrm{O}_{3}$ (1 $\mathrm{mL}, 0.5 \mathrm{mM}$ ) y se dejó reaccionar por un periodo de 2 horas a $19-20^{\circ} \mathrm{C}$ en agitación constante. Pasado este tiempo, se tomó una alícuota de la muestra para la caracterización mediante UV-Vis-NIR y TEM.

\subsection{FUNCIONALIZACIÓN DE AUNTS}

Los nanosensores aptaméricos (AuNTs@Apt) se prepararon a partir de $500 \mu \mathrm{L}$ de los AuNTs recién sintetizados, a los que se les adicionó SDS ( $2.5 \mu \mathrm{L}, 10 \%)$. A continuación, para asegurar una adecuada funcionalización se añadieron, en orden, los siguientes reactivos: tampón de fosfato $\mathrm{pH} 8(100 \mu \mathrm{L}, 100 \mathrm{mM})$, solución de aptámero de AFB1 $(2.5 \mu \mathrm{L}, 200 \mu \mathrm{M})$ y 4 adiciones de solución de $\mathrm{NaCl}(25 \mu \mathrm{L}$ en cada adición, 1 M). Finalmente, una solución acuosa de MPA ( $2 \mu \mathrm{L}, 11.5$ $\mathrm{mM}$ ) fue agregada a la mezcla para aumentar la estabilidad de las nanopartículas al unirse a la superficie libre de las nanoestructuras.

Luego de completar el proceso de funcionalización, el nanosensor (AuNTs@Apt@MPA) fue centrifugado 3 veces a $6000 \mathrm{rpm}$ por 10 minutos. A continuación, se resuspendió el sensor en un volumen final de $1 \mathrm{~mL}$ con agua ultrapura.

\subsection{DETECCIÓN DE AFB1}

Después de la purificación, se diluyeron $100 \mu \mathrm{L}$ del nanosensor en una solución acuosa que contenía uno de los 4 tampones preparados (ver detalles en Tabla I) en agitación constante por 5 minutos. Posteriormente, se agregó una solución de AFB1 y luego de 10 minutos se midió el espectro UV-Vis-NIR de la solución resultante. Para construir las curvas de calibración LSPR se procesaron los datos en el programa OriginPro (OriginLab, Massachusetts, USA) aplicando un suavizado (orden 5, 51 ptos) y normalizando los espectros a $400 \mathrm{~nm}$. Finalmente, se relacionaron las concentraciones de AFB1 (o - 780 ppbs) con la diferencia de la integral espectral en el rango de $630-$ $1000 \mathrm{~nm}$ en ausencia y presencia de la micotoxina $\left(\right.$ Área $=$ Área $_{0}-$ Área $\left._{\mathrm{AFB} 1}\right)$.

Para el análisis multivariante por regresión de mínimos cuadrados parciales (PLS) se utilizó el programa Solo (Eigenvector, Seattle, USA). Este análisis permitió comparar la concentración de soluciones de $A F B 1$ reales $\left([A F B 1]_{\text {real }}\right)$ con las concentraciones de AFB1 predichas por el modelo matemático $\left([\mathrm{AFB} 1]_{\mathrm{pred}}\right)$. El preprocesamiento utilizado fue línea base (mínimos cuadrados ponderados automáticos, orden 2), suavizado (orden 2, 15 ptos) y ponderación de mínimos cuadrados generalizados (GLSW, 0.02), en el rango de 500 a $1000 \mathrm{~nm}$.

A

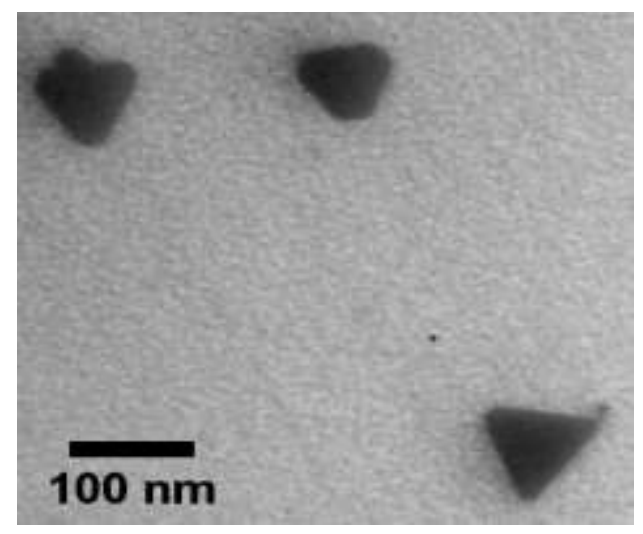

B

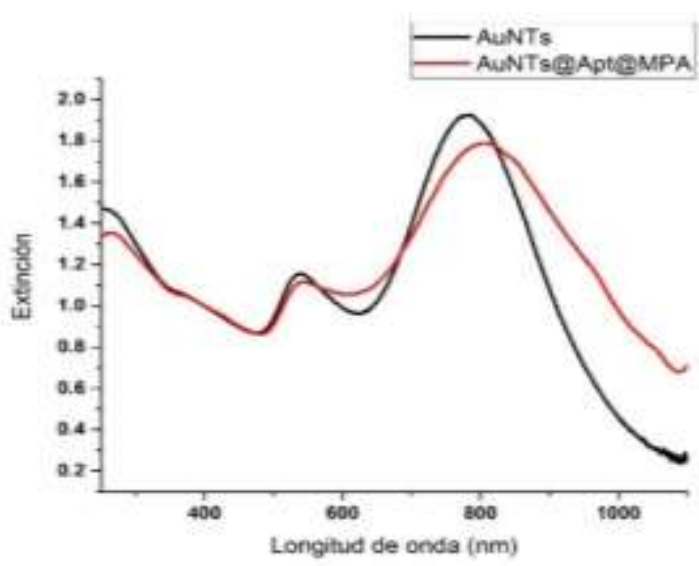

Fig. 1. Caracterización de los AuNTs: A) micrografía TEM de los AuNTs sin funcionalizar, y B) espectros de extinción UVVis-NIR de AuNTs sin funcionalizar y AuNTs@Apt@MPA. 
TABLA I
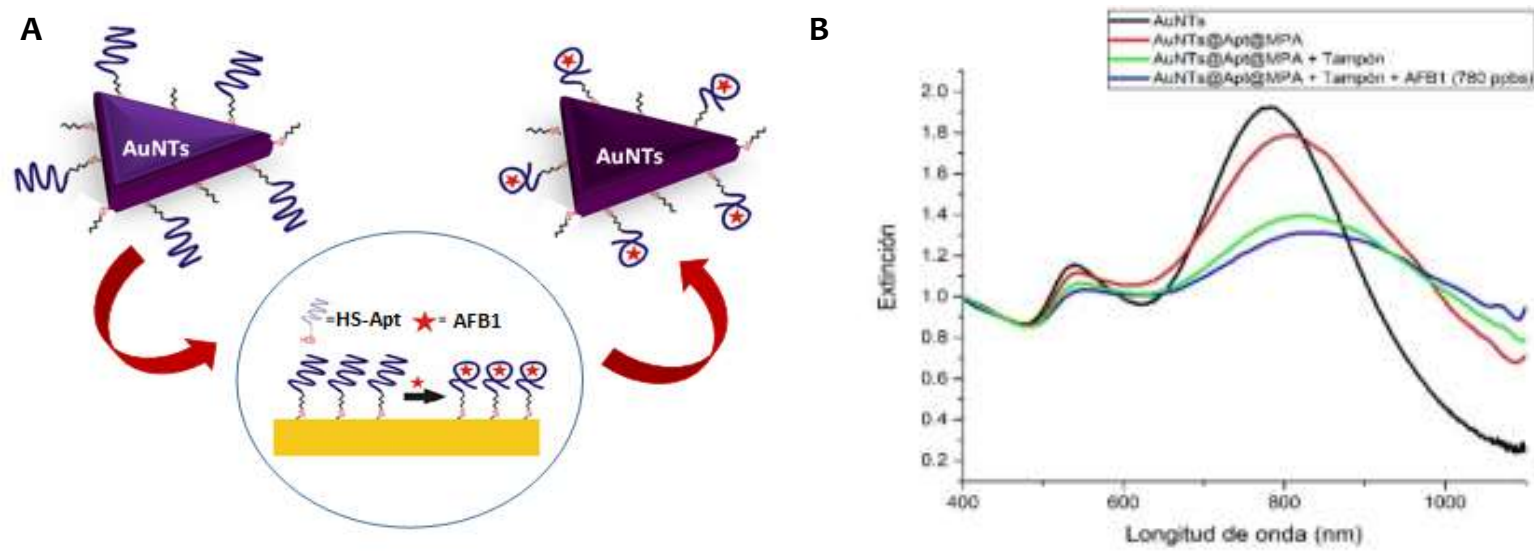

Fig 2. A) Esquema del proceso de detección de la AFB1 utilizando el nanosensor AuNTs@Apt@MPA. B) Espectros de extinción de los AuNTs, AuNTs@Apt@MPA y AuNTs@Apt@MPA con el tampón de PBS con MgCl $_{2}$ en ausencia y presencia de AFB1 (780 ppbs).

TABLA I

Información sobre las curvas de calibración LSPR para AFB1 (0-780 ppbs) utilizando el sensor AuNTs@Apt@MPA en diferentes tampones y solventes.

\begin{tabular}{|c|c|c|c|}
\hline Código del tampón & Sistema de solventes & Pendiente & $\mathrm{R}^{2}$ \\
\hline PBS pH 7.5 & $10 \mathrm{mM} \mathrm{K}_{2} \mathrm{HPO}_{4}, 2 \mathrm{mM} \mathrm{KH}_{2} \mathrm{PO}_{4}, 137 \mathrm{mM} \mathrm{NaCl}, 2.7 \mathrm{mM} \mathrm{KCl}, 2 \mathrm{mM} \mathrm{MgCl}, \mathrm{PH} 7.5+0.1 \% \mathrm{MeOH}$ & -0.0324 & 0.98101 \\
\hline PBS PH 7.5 Con $10 \% A C N$ & $10 \mathrm{mM} \mathrm{K}_{2} \mathrm{HPO}_{4}, 2 \mathrm{mM} \mathrm{KH}_{2} \mathrm{PO}_{4}, 137 \mathrm{mM} \mathrm{NaCl}, 2,7 \mathrm{mM} \mathrm{KCl}, 2 \mathrm{mM} \mathrm{MgCl}, \mathrm{PH} 7.5+0.1 \% \mathrm{MeOH}+10 \% \mathrm{ACN}$ & 0.03356 & 0.64739 \\
\hline PB5 pH 7.5 con $1.95 \%$ MeOH & $10 \mathrm{mM} \mathrm{K}_{2} \mathrm{HPO}_{4}, 2 \mathrm{mM} \mathrm{KH}_{2} \mathrm{PO}_{4}, 137 \mathrm{mM} \mathrm{NaCl}, 2.7 \mathrm{mM} \mathrm{KCl}, 2 \mathrm{mM} \mathrm{MgCl}, \mathrm{PH} 7.5+1.95 \% \mathrm{MeOH}$ (no lineal) & - & - \\
\hline PB5 pH 6.5 & $10 \mathrm{mM} \mathrm{K}_{2} \mathrm{HPO}_{4}, 2 \mathrm{mM} \mathrm{KH}_{2} \mathrm{PO}_{4}, 137 \mathrm{mM} \mathrm{NaCl}, 2.7 \mathrm{mM} \mathrm{KCl}, 2 \mathrm{mM} \mathrm{MgCl}, \mathrm{PH} 6.5+0.1 \% \mathrm{MeOH}$ & -0.03386 & 0.87635 \\
\hline TRIS pH 7 & $10 \mathrm{mM}$ Tris, $120 \mathrm{mM} \mathrm{NaCl}, 20 \mathrm{mM} \mathrm{CaCl},+5 \mathrm{mM} \mathrm{KCl} \mathrm{pH} 7+0.18 \mathrm{MeOH}$ & -20.66751 & 0.97187 \\
\hline TRIS pH 8.5 & $10 \mathrm{mM}$ Tris, $120 \mathrm{mM} \mathrm{NaCl}$, $20 \mathrm{mM} \mathrm{CaCl}+5 \mathrm{mM} \mathrm{KCl}$ pH $8.5+0.1 \% \mathrm{MeOH}$ & -0.14989 & 0.55869 \\
\hline
\end{tabular}

\subsection{PREPARACIÓN DE EXTRACTOS DE KIWICHA}

Se pesaron 2.0 gramos de kiwicha en un tubo de centrifuga de $50 \mathrm{~mL}$ y se le agregaron $10 \mathrm{~mL}$ de la solución de extracción $\mathrm{MeOH}: \mathrm{H} 2 \mathrm{O}$ (7:3). La suspensión obtenida fue sonicada durante 15 minutos a $10{ }^{\circ} \mathrm{C}$, y a continuación fue centrifugada durante 30 minutos a $10^{\circ} \mathrm{C}$ y 7830 RPM. Luego se extrajo el sobrenadante y se pasó por una jeringa con filtro de fibra de vidrio, recogiéndose finalmente $7.5 \mathrm{~mL}$ de extracto.

\section{RESULTADOS Y DISCUSIÓN}

\subsection{CARACTERIZACIÓN DE AUNTS}

La caracterización de los nanosensores aptaméricos se llevó a cabo utilizando un microscopio electrónico de

transmisión y un espectrofotómetro UV-Vis NIR. Los nanotriángulos tuvieron un tamaño de $52 \pm 16 \mathrm{~nm}$ de lado y $9 \pm 1 \mathrm{~nm}$ de borde (Fig. $1 \mathrm{~A}$ ), y un espectro de extinción de la banda localizada a $785 \pm 30 \mathrm{~nm}$. Luego de la funcionalización, se obtuvo un nuevo espectro de extinción, gracias al cual se pudo observar un desplazamiento de la banda plasmónica de 4-10 nm, indicativo de la unión entre los AuNTs y el aptámero. (Fig. 1B).

\subsection{DETECCIÓN DE AFB1 UTILIZANDO AUNTS@APT@MPA}

El proceso de detección de la AFB1 se llevó a cabo siguiendo el esquema mostrado de la Fig. $2 \mathrm{~A}$ y analizando la curva de calibración (Área Promedio vs [AFB1]). Gracias a los espectros de extinción obtenidos se pudieron observar los cambios en el área de la banda plasmónica por el sensor al momento de entrar en contacto con la micotoxina (AFB1). La Fig. 2B muestra la diferencia más drástica, donde la banda plasmónica del sensor AuNTs@Apt@MPA se desplaza a mayores longitudes de onda debido a que el aptámero interacciona con la micotoxina $([\mathrm{AFB} 1]=780$ ppbs), induciendo un cambio conformacional en la estructura del aptámero que genera una alteración en los alrededores de los nanotriángulos.

Para optimizar el proceso de detección, los ensayos de LSPR fueron evaluados utilizando diferentes solventes orgánicos como $\mathrm{MeOH}$ y ACN, los cuales son utilizados comúnmente en los procesos de extracción [17]. Además, se utilizaron diferentes tampones, como los descritos en la Tabla I, los cuales han sido reportados en la literatura como medios adecuados 
para favorecer la formación del complejo aptameromicotoxina debido a sus relativamente bajas constantes de disociación (KD) y, posiblemente, a la presencia de cationes $\mathrm{Ca}_{2}+$ y $\mathrm{Mg}_{2+}$, que permiten estabilizar la nueva conformación del aptámero [2], [16].

Cada ensayo fue realizado por triplicado y en tres diferentes días, utilizando a su vez diferentes lotes de nanosensores. Los espectros obtenidos fueron analizados siguiendo el protocolo descrito en la metodología y utilizando la región comprendida entre 630-1000 nm para concentraciones de AFB1 de 0 a 780 ppbs. Los resultados obtenidos se encuentran resumidos en la Tabla I, donde se observó que la curva de calibración obtenida (Fig. 3) con el tampón PBS pH 7.5 fue la que evidenció mejor sensibilidad $\left(R^{2}=0.98101\right.$, límite de detección de 234 ppbs calculado a partir de 3 veces la desviación estándar del segundo punto de la curva).

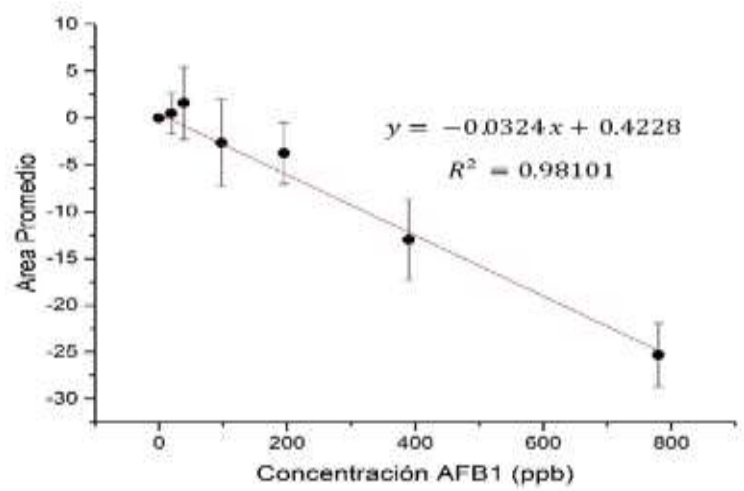

Fig. 3. Curva de calibración LSPR obtenida con el tampón de PBS pH 7.5 para un rango de concentración de $0-780$ ppbs de AFB1.

Teniendo en consideración que el tampón de PBS pH 7.5 tuvo los mejores resultados, los datos obtenidos a partir de sus ensayos fueron analizados también mediante el método de regresión de mínimos cuadrados parciales (PLS). Asimismo, se analizaron 2 sistemas de solventes adicionales preparados a partir del mismo tampón de PBS pH 7.5, el primero de ellos con $1.95 \%$ de $\mathrm{MeOH}$ y el segundo con $10 \%$ de ACN. Los resultados del análisis por PLS se presentan en la Tabla II en donde, en concordancia con lo obtenido previamente, se observó que el modelo matemático de PLS para el tampón de PBS pH 7.5 era adecuado para la detección de AFB1. En los tres casos se obtuvieron resultados muy similares, lo que demuestra la robustez del método. Por ejemplo, en presencia de $0.1 \%$ de $\mathrm{MeOH}$ se obtuvo un $\mathrm{R}^{2}$ de validación de 0.993 y un RMESCV de 21.9597. La curva de calibración obtenida para este tampón se muestra en la Fig 4.

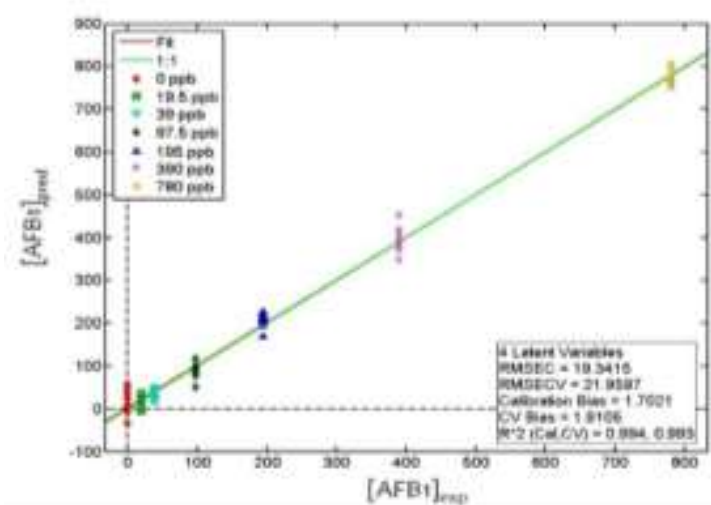

Fig. 4. Análisis PLS para el sistema de detección de AFB1 (o-780 ppbs) utilizando el nanosensor AuNTs@Apt@MPA en la solución tampón de PBS pH 7.5.

TABLA II

Información sobre las curvas de calibración LSPR para AFB1 obtenidas utilizando el sensor AuNTs@Apt@MPA en tres diferentes tapones a través de (PLS).

\begin{tabular}{l|ll}
\hline Tampones utilizados & R2 validación & RMSECV \\
\hline PBS pH 7.5 & 0.993 & 20.9597 \\
PBS pH 7.5 con 10\% ACN & 0.970 & 21.9561 \\
PBS pH 7.5 con 1.95\% MeOH & 0.974 & 20.4423 \\
\hline
\end{tabular}

\subsection{Detección de AFB1 en muestras reales de kiwicha}

Una vez optimizado el nanosensor y habiendo seleccionado el sistema de solventes basado en el tampón de PBS pH 7.5, se decidió evaluar la respuesta del sensor AuNTs@Apt@MPA en muestras reales de kiwicha. Para ello se evaluó la respuesta del nanosensor con extractos de kiwicha contaminados con una cantidad conocida de AFB1 ( 0 - 780 ppbs). Los ensayos se realizaron en tres días diferentes, por triplicado y utilizando diferentes lotes de nanosensores, siguiendo el protocolo general de detección de AFB1 para evaluar el posible efecto de la matriz orgánica presente en los extractos.

Los espectros de extinción fueron analizados en el rango de 630 - $1000 \mathrm{~nm}$ obteniéndose una curva para las concentraciones de AFB1 de o a 532 ppbs cuya pendiente fue de -0.03865 con un R2 de 0.98226 . Como se observa en la Fig.5, al comparar los resultados obtenidos con los extractos de kiwicha contaminados con la curva obtenida con las soluciones estándar en el tampón de PBS pH 7.5, se aprecian pendientes muy similares entre sí, sugiriendo que es posible determinar el contenido de AFB1 en extractos de kiwicha con nuestro método de detección, y que este sistema de detección es lo suficientemente robusto, en presencia de una matriz compleja como el extracto de kiwicha. 


\section{CONCLUSIONES}

- En esta investigación se logró obtener un nanosensor aptamérico capaz de detectar AFB1 en el rango de los ppbs, de manera sencilla, accesible y rápida. El sensor fue evaluado en diferentes sistemas de solventes obteniéndose una mejor curva de calibración para el buffer de PBS pH 7.5 con un $\mathrm{R}^{2}$ de 0.98101 en comparación a los otros sistemas de solventes evaluados. Además, el análisis multivariante mediante el método de PLS permitió obtener un modelo adecuado para la detección de AFB1 basado en el tampón de PBS con $\mathrm{MgCl} 2$ con un límite de detección de 22 ppbs. Finalmente, al probar el sensor en una matriz compleja como la kiwicha se observó que los resultados obtenidos con los extractos contaminados y los valores de la curva de calibración con las soluciones estándar en el tampón de PBS pH 7.5 poseen pendientes similares $\quad(-0.0324$ y -0.03865 , respectivamente), permitiéndonos concluir que la matriz orgánica compleja proveniente de los extractos no afecta en gran medida los resultados obtenidos. Por tal motivo, los nanosensores aptámericos desarrollados en esta investigación son los primeros pasos para la creación de un sistema de detección de AFB1 sencillo y rápido, aun cuando las muestras estén inmersas en una matriz compleja como la de los extractos de kiwicha.

\section{AGRADEDIMIENTOS}

Este trabajo fue financiado por el Programa Nacional de Innovación Agraria (N021-2016-INIAPNIA/UPMSI/IE) y la Dirección de Gestión de la investigación de la PUCP (CAP2017-431). Las imágenes TEM se obtuvieron gracias a Innóvate Perú ( ${ }^{\circ} 281$ INNOVATEPERU-EC-2017).

\section{REFERENCIAS}

[1] A. García-moraleja, G. Font, J. Mañes, and E. Ferrer, "Simultaneous determination of mycotoxin in commercial coffee," Food Control, vol. 57, pp. 282-292, 2015.

[2] J. A. Cruz-Aguado, "Determination of Ochratoxin A with a DNA Aptamer," pp. 10456-10461, 2008.

[3] J. W. Bennett and M. Klich, "Mycotoxins" Society, vol. 16, no. 3, pp. 497-516, 2003.

[4] S. Marin, A. J. Ramos, G. Cano-Sancho, and V. Sanchis, "Mycotoxins: Occurrence, toxicology, and exposure assessment," Food Chem. Toxicol., vol. 60, pp. 218-237, 2013.

[5] World Health Organization, Prevention and Reduction of Food and Feed Contamination. Food and Agriculture Organization of the United Nations (FAO), 2012.

[6] Norma UNE-EN 14123:2008 para la “Determinación de aflatoxina B1 y de la suma de las aflatoxinas B1, B2, G1 y G2 en avellanas, cacahuetes, pistachos, higos y pimentón molido. Método por cromatografía líquida de alta resolución con transformación química postcolumna y purificación en columna de inmunoafinidad".

[7] Y. Luan et al., "Rapid Visual Detection of Aflatoxin B1 by LabelFree Aptasensor Using Unmodified Gold Nanoparticles," J. Nanosci. Nanotechnol., vol. 15, no. 2, pp. 1357-1361, 2014.

[8] T. C. Chiu and C. C. Huang, Aptamer-functionalized nanobiosensors, vol. 9, no. 12. 2009.

[9] A. Rhouati, C. Yang, A. Hayat, and J. Marty, "Aptamers: A Promising Tool for Ochratoxin A Detection in Food Analysis," pp. 1988-2008, 2013.

[10] B. C. Galarreta, M. Tabatabaei, V. Guieu, E. Peyrin, and F. Lagugné-Labarthet, "Microfluidic channel with embedded SERS 2D platform for the aptamer detection of ochratoxin A," Anal. Bioanal. Chem., vol. 405, no. 5, pp. 1613-1621, 2013.

[11] L. K. Lagos, L. Veliz, Y. Hernandez, and B. C. Galarreta, "Labelfree SERS and LSPR gold nanoaptasensors of mycotoxins in solution: solvent assessment,"Latin American Optics and Photonicsonference, p.Tu2C.2,2018.

[12] J. Ko, C. Lee, and J. Choo, "Highly sensitive SERS-based immunoassay of aflatoxin B1 using silica-encapsulated hollow gold nanoparticles,” J. Hazard. Mater., vol. 285, pp. 11-17, 2015.

[13] S. Alex and A. Tiwari, "Functionalized Gold Nanoparticles: Synthesis, Properties and Applications-A Review," J. Nanosci. Nanotechnol., vol. 15, no. 3, pp. 1869-1894, 2015.

[14] S. Eustis and M. A. El-Sayed, "Why gold nanoparticles are more precious than pretty gold: Noble metal surface plasmon resonance and its enhancement of the radiative and nonradiative properties of nanocrystals of different shapes," Chem. Soc. Rev., vol. 35, no. 3, pp. 209-217, 2006.

[15] M. Hosseini, H. Khabbaz, M. Dadmehr, M. R. ez. Ganjali, and J. Mohamadnejad, "Aptamer-based Colorimetric and Chemiluminescence Detection of Aflatoxin B1 in Foods Samples," Acta Chim. Slov., vol. 62, no. 3, pp. 721-728, 2015.

[16] X. Geng, D. Zhang, H. Wang, and Q. Zhao, "Screening interaction between ochratoxin A and aptamers by fluorescence anisotropy approach," Anal. Bioanal. Chem., vol. 405, no. 8, pp. 2443-2449, 2013. [17] B. Pelaz, "Tailoring the Synthesis and Heating Ability of Gold Nanoprisms for Bioapplications," Langmuir, vol. 28, no. 24, pp.89688970, 2012.

[18] Y. Hernández García, J. R. Sifuentes, A. S. Saldaña, and B. C. Galarreta, "Estudio de los factores determinantes para el desarrollo de sensores SERS efectivos y económicos," Tecnia, vol. 28, no. 2, 2018.

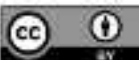
compartidos a través de la licencia Creative Commons: CC BY 4.0. Permisos lejos de este alcance pueden ser consultados a través del correorevistas@uni.edu.pe 